\title{
Optimization of Performance Metrics of LAR IN AD-HOC NETWORK
}

\author{
Neelesh Gupta ${ }^{1}$ and Roopam Gupta ${ }^{2}$ \\ ${ }^{1 .}$ Research Scholar, UIT, RGPV, Bhopal (M.P.)-India \\ neelesh.9826@gmail.com \\ 2. Dept. of IT, UIT, RGPV, Bhopal (M.P.)-India \\ roopamgupta@rgtu.net
}

\begin{abstract}
Routing in Mobile Ad-Hoc Network (MANET) is a crucial task due to highly dynamic network environment. In latest years, several routing protocols have been implemented. In recent developments, position-based routing protocols exhibit better scalability performance and robustness against frequent topological changes such as, Location-Aided Routing (LAR) protocol. In this paper, developments of performance-metrics using LAR have been reported. Using these developments, LAR protocol has been found to be better than other protocols like DSR in MANETs of different scenario. This is because of already available location information of the nodes in network while it is based upon source routing as a DSR. Via LAR, performance metrics Packet Delivery Fraction (PDF), routing overhead, End to End (E2E) Delay, Non-Routing Load (NRL) and Number of lost (dropped) packets during route discovery can be optimized in dynamic Ad-Hoc networks.
\end{abstract}

\section{KEYWORDS}

MANETs, LAR, DSR, Performance Metrics.

\section{INTRODUCTION}

Mobile Ad-Hoc Network is an infrastructure less, self-organizing, self-configuring, selfmaintaining network designed by a set of wireless mobile nodes, where all the mobile hosts take part in the process of forwarding packets. These are highly applicable in Military Networks, Personal Area Networks, Home Networks, Wireless Sensor Networks, and Inter-Vehicle Communication. Each node in the network also acts as a router, forwarding data packets for other nodes. A central challenge in the design of Ad-Hoc networks is the development of dynamic routing protocols. A routing protocol is needed whenever a packet needs to be transmitted to a destination via number of nodes and numerous routing protocols have been proposed for Ad-Hoc networks. Several routing protocols have been planned to achieve a particular level of routing operation for MANET. The routing protocols are divided into several categories. The most popular classification is between Topological and Position-based routing Protocols. Under Topological-based proactive and reactive protocols come. In proactive a source node wants to transmit the data from $S$ to $D$, it searches the routing table to find a destination node match. Destination Sequenced Distance Vector (DSDV), Wireless Routing Protocol (WRP), Cluster Switch Gateway Routing (CGSR), Source Tree Adaptive Routing Protocol (STAR) is the examples of proactive protocols. The main drawback of these protocols is that the maintenance of unused paths may occupy a significant part of the available bandwidth if the topology of the network changes frequently. In reactive, the routes are discovered only when the source needs to transmit the data. Dynamic Source Routing Protocol (DSR), Ad-Hoc On-Demand Distance-Vector Routing Protocol (AODV), Temporally-Ordered

DOI : 10.5121/ijwmn.2012.4306 
Routing Algorithm (TORA) is the reactive routing protocols [7] [9]. These reduce the burden on the network when only a small subset of all available routes is in use at any time. Further position-based routing protocols eliminate some of the inherent limitations of topology-based routing by using additional location information. The routing decision at each node is then based on the destination's position contained in the packet and the position of the forwarding node's neighbours. Position-based routing does not require the establishment or maintenance of routes [9]. There are three main packet forwarding schemes in position-based routing: Greedybased, Restricted-directional flooding and Hierarchical approaches. For the first two, a MH forwards a given packet to one (greedy-forwarding) or more (restricted directional flooding) one-hop neighbours. The third forwarding strategy forms a hierarchy in order to scale to a large number of MHs. Greedy-based Routing Protocols do not establish and maintain paths from S to D. In this packet sender node includes approximate position of the recipient in packet. MFR, GPSR are examples. In Restricted Directional flooding-based Routing Protocols source floods data packets in a restricted geographical area towards the direction of destination. Instead of selecting a single node as the next hop several nodes participate in forwarding the packet in order to increase the probability of finding the shortest path. Examples are Location-Aided Routing Protocol (LAR), Distance Routing Effect Algorithm for Mobility (DREAM) routing protocols. Hierarchal-based Routing Protocols form a hierarchy in order to scale to a large number of MHs. Complexity of the routing algorithm can be reduced tremendously by establishing some form of hierarchy also. Examples are GRID \& TERMINODES.

LAR is source routing protocol, as a DSR. Initially it starts flooding in all the directions by the source after expecting the destination the routing is so easy in network that will be in the direction of the destination. This is the basic principle of directional-restricted position based location-aided routing in MANET. In this paper, optimizations of Ad-Hoc network performance metrics are revealed via restricted directional flooding-based LAR scheme. In the second section, related work is presented. Overview of DSR and LAR protocols is shown in the next section. In the fourth section simulation results on DSR and LAR performance discussion and displays are shown. The last section concludes the paper.

\section{RELATED WORK}

Earlier several researchers had been devoted their contribution for the evaluation of various routing protocols. Das S. et al. [3] compared performance of two on-demand routing protocols (AODV \& DSR) for Ad-Hoc networks. The general observation from the simulation is for the application oriented metrics such as delay and throughput. DSR outperforms than AODV in less stressful situations (smaller number of nodes and lower load/mobility). However, AODV outperforms than DSR in more stressful situations (more load and higher mobility). However, DSR consistently generates less routing load than AODV. Mauve M. et al. [9] presented an overview of MANET routing protocols that make forwarding decisions based on the geographical position of a destination packet. They also provided a qualitative comparison of position-based like a DREAM, LAR, GLS and Greedy routing approaches. They also finally concluded that LAR and DREAM scheme can be used where small number of packets to be need transmitted very reliably in the network.

E.Ahver et al. [6] compared the performance of three routing protocols for Ad-Hoc network: DSR, AODV and LAR-1. Their evaluation is based on energy consumption in MANETs. Performance analysis is examined using varying network load, mobility and network size of the network. DSR consumes the least energy for low density networks. LAR-1 for high density networks is much better than others. Therefore, LAR-1 is a good protocol for high density AdHoc networks. Y.B. Ko. et al. [8] proposed an optimization to route-discovery known as LAR protocol that uses GPS for location of all mobile nodes in networks. They suggested LAR 
approach to utilize location information using GPS to improve performance of routing protocols for Ad-Hoc networks. LAR protocols limit the search for a new route to a smaller "request zone" of the Ad-Hoc network. This results in a significant reduction in the number of routing messages and also presented two LAR algorithms to determine the request zone optimizations suggestions to algorithms. Qabajeh L. et al. [11] explained a qualitative comparison of the existing geographic routing protocols that make forwarding decisions based on the geographical position of a packets destination. Advantages of all position-based strategy are illustrated in this research paper. Finally they identified a number of research opportunities which could lead to further improvements in position-based Ad-Hoc routing also. Camp T. et al. [2] clarified the performances of LAR and DREAM routing protocols and compared the both with DSR in MANETs. Their analysis produces that via adding location information to DSR (like LAR) network load and PDF both are improved. The project aim of David D. et al. [4] was to test routing performance of four different routing protocols (AODV, DSR, LAR 1 and ZRP) as a function of network and area size. This describes the different routing protocols, the experiment setup and finally presents the simulation results. AODV outperforms than DSR. LAR-1 is even better than AODV up to 200 nodes in terms of delivery ratio and routing overhead due to the geographical information of the node. Despite the popularity of several protocols, research efforts have not focused in evaluating their performance in large-scale wireless networks. This greatly affects the network efficiency, since it necessitates frequent exchange of routing information. Broustic I. et al. [1] presented the behaviour of the DSR, AODV, TORA and LAR protocols in large-scale MANETs. They concluded that DSR scales well in terms of packet delivery fraction but suffers an important increase of end-to-end delay, as compared to its performance achieved in small scale topologies. LAR appears to scale very well in terms of all metrics employed.

\section{OUTLINE OF DSR AND LAR ROUTING PROTOCOL}

DSR: The Dynamic Source Routing (DSR) is one of the examples of an on-demand (reactive) routing protocol that is based on the concept of source routing. It is designed especially for use in multi-hop Ad-Hoc networks of mobile nodes. DSR is composed of the two mechanisms of route-discovery and route maintenance, which work together to allow nodes to discover and maintain source routes to arbitrary destinations in the network. When a node in the Ad-Hoc network attempts to send a data packet to a destination for which it does not know the route, it uses a route discovery process to dynamically determine a route. Route discovery works by flooding the network with route request (RREQ) packets. Each node receiving an RREQ rebroadcasts it, unless it is the destination or it has a route to the destination in its route cache. Such a node replies to the RREQ with a route reply (RREP) packet that is routed back to the original source [5] [6].

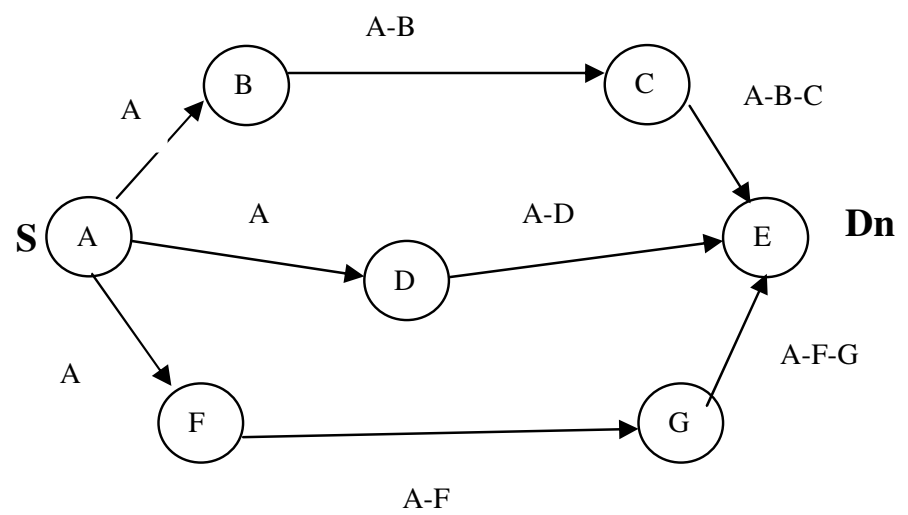

Figure.1.a RREQ Pаскеє коute Discovery Mechanism 


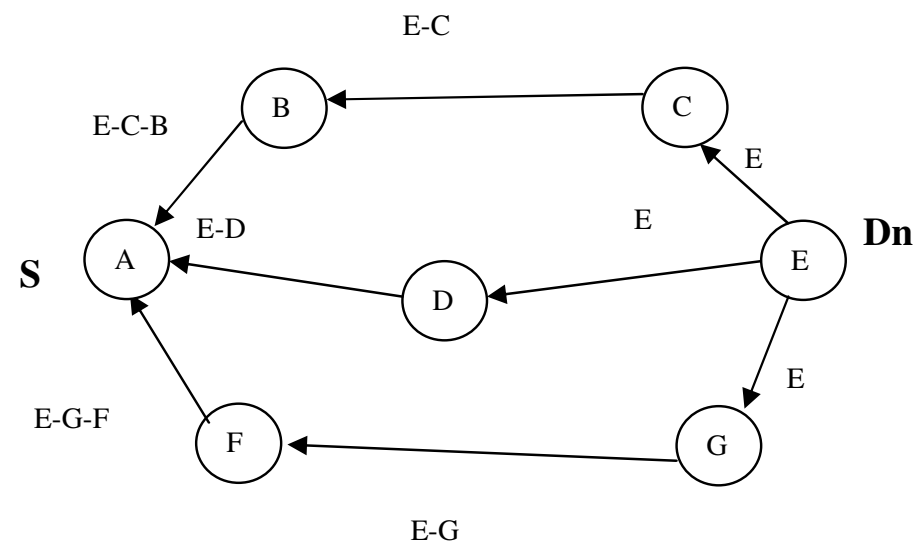

Figure.1.b RREP Packet Route Discovery Mechanism

\section{Figure1. DSR Route Discovery Mechanism}

LAR: LAR is source routing protocol, as a DSR. Initially it starts flooding in all the directions by the source after expecting the destination the routing is so easy in network that will be only in the direction of the destination. It sends the location information in all packets to decrease the routing overhead in future route discovery process in the network. It uses the location information by using GPS. Less routing overhead with LAR can be achievable by limiting the search space for the desired route to a destination into minor request regions in the network. LAR uses a request zone that is rectangular in shape. Consider a node $S$ (Source) that needs to find a route to node $\mathrm{D}$ (Destination). Assume that node $\mathrm{S}$ knows that node $\mathrm{D}$ was at location $(\mathrm{Xd}, \mathrm{Yd})$ at time $\mathrm{t}_{0}$. At time $\mathrm{t}_{1}$, node $\mathrm{S}$ initiates a new route discovery for destination $\mathrm{D}$. It assumes that node $S$ also knows the average speed $v$ with which $D$ can move. Using this, node $S$ defines the expected zone at time $t_{1}$ to be the circle of radius $R=v\left(t_{1}-t_{0}\right)$ centered at location $(\mathrm{Xd}, \mathrm{Yd})$. In figure 2.a, $\mathrm{t}_{1}-\mathrm{t}_{0}$ is the elapsed time between two successive route requests from the source node. When a node receives a route request, it discards the request if the node is not within the request region. For instance, in Fig.2.b, if node $M$ receives the route request from another node, node $\mathrm{M}$ forwards the request to its neighbours, because $\mathrm{M}$ is within the rectangular request zone. However, when node $\mathrm{N}$ receives the route request, node $\mathrm{N}$ discards the request, as node $\mathrm{N}$ is not within the request zone [6], [7] and [9].

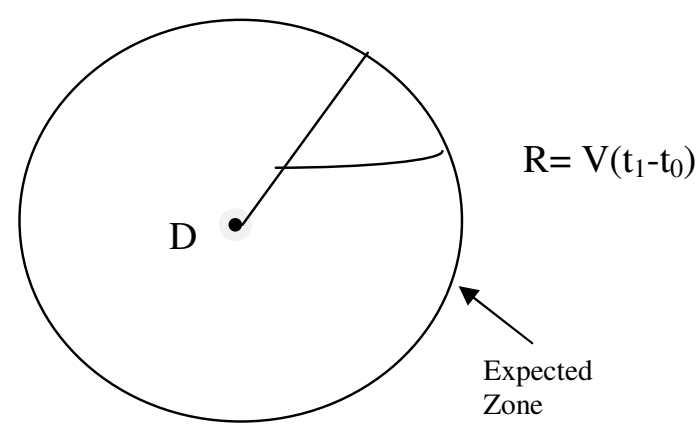

Figure2.a Expected Zone 


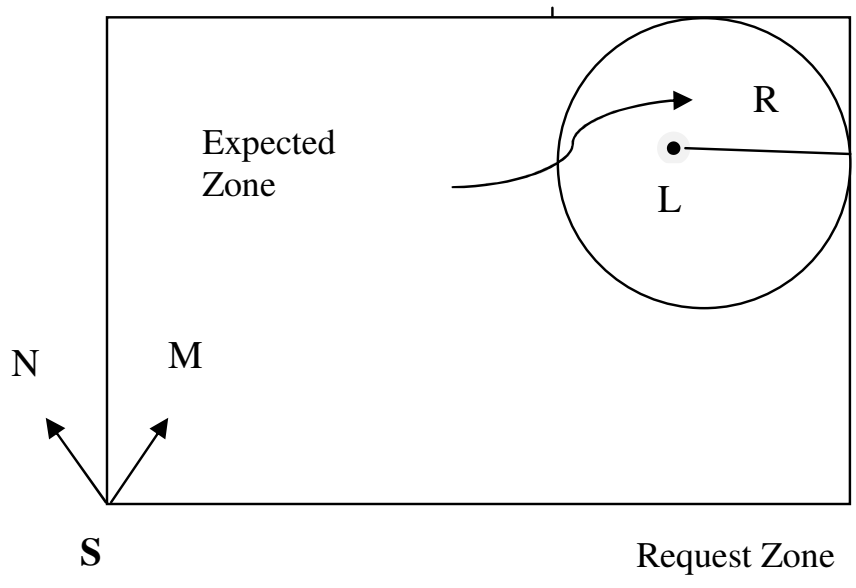

Figure 2.b LAR-1 Request Region

Figure 2. LAR-1 Scheme

DSR and LAR both use source routing in network but difference is that LAR at first start routing as a DSR but after expecting the destination packets are flooded in the direction of destination only. As a result the routing overhead is to be reduced and better performance of LAR protocol is obtained.

\section{SIMULATION RESULTS AND DISCUSSION}

The simulation study was conducted in the "Network Simulator" (NS2) environment and used the Ad-Hoc networking extensions provided by CMU [10]. All simulations were performed on Intel (R) core (TM) i3 CPU, $2.3 \mathrm{GHZ}, 3072 \mathrm{MB}$ of RAM running on Inspiron N5010 configuration. These include physical, data link and medium access control layer models. The Distributed Coordination Function (DCF) of IEEE 802.11 is used to model the contention of nodes for the wireless medium. The radio model uses characteristics similar to Lucent's WaveLAN direct sequence spread spectrum radio. The source-destination pairs were spread randomly over the network. Constant bit rate (CBR) traffic sources are used in simulation. The size of these packets is 512 bytes with transport agents TCP \& UDP. The random waypoint mobility model has been used in a rectangular filed area with different number of mobile nodes. This model based on random waypoints and random speeds that includes pause times between changes in destination and speed. In this model, a mobile node moves from its current location to a randomly chosen new location within the simulation area using a random speed uniformly distributed between the maximum and minimum speed of the simulation. The simulations were run for 100 seconds. The number of nodes (n) in simulated Ad-Hoc network is 12, 24, 36, 48 and situated in a 800x600 square meter region having a transmission range of 550m. In this simulation, each node starts its journey from a random location to a random destination with a randomly chosen speed (uniformly distributed average speed of $20 \mathrm{~m} / \mathrm{sec}$ ). Once the destination is reached, another random destination is chosen after a pause time. Pause time is taken in the interval of 20 seconds during simulation. This model is often simplified by using a uniformly distributed speed. 
Table 1. Simulation Environment

\begin{tabular}{|l|l|}
\hline Network Parameter & Value \\
\hline Simulator & NS-2 \\
\hline Simulation time & 100 Seconds \\
\hline Transmission range & $550 \mathrm{~m}$ \\
\hline Node movement model & Random way point \\
\hline Protocols studied & DSR and LAR \\
\hline Simulation area & $800 \times 600$ \\
\hline Bandwidth & $2 \mathrm{Mb} / \mathrm{s}$ \\
\hline Traffic type & CBR \\
\hline Transport Agents & TCP and UDP \\
\hline Pause Time & $0-100 \mathrm{~s}$ in steps of $20 \mathrm{~s}$ \\
\hline Average Node Speed & $20 \mathrm{~m} / \mathrm{s}$ \\
\hline Packet Size & 512 bytes \\
\hline
\end{tabular}

\subsection{Performance Metrics}

The following performance metrics are considered for evaluation:

(a) Packet Delivery Fraction (PDF): The ratio of the data packets delivered to the destinations to packets generated by the sources. It specifies the packet loss rate which limits the maximum throughput of the network. For efficient routing protocol PDF should be more.

(b) End-to-End Delay (E2E): End-to-End Delay indicates how long time is taken for a packet to travel from the source to the destination during routing in networks. This includes all possible delays caused by buffering during route discovery latency, queuing at the interface queue, retransmission delays at the MAC, propagation and transfer times.

(c) Normalized Routing Load (NRL): The number of routing packets transmitted per data packet delivered at the destination. It is concerned with number of routing packets.

(d) Routing Overhead: The routing overhead describes how many routing packets for route discovery need to be sent in order to propagate the data packets. It is an important measure for the scalability of a protocol.

\subsection{Simulation Results and Parameters}

The simulation results are revealed in the following section in the form of line graphs. Graphs show comparison between the two (DSR and LAR-1) protocols by varying different numbers of nodes on the basis of the above-mentioned performance metrics as a function of pause time and number of nodes.

\subsubsection{Results-}

After simulating in NS-2 simulator value of PDF increases using LAR by $0.56 \%$, reduces routing overhead or RLOAD, E2E Delay and Dropped packets by $9.25 \%, 17.8 \%$ and $28.6 \%$ respectively compared to DSR protocol. NRL for both is almost equal. 
International Journal of Wireless \& Mobile Networks (IJWMN) Vol. 4, No. 3, June 2012

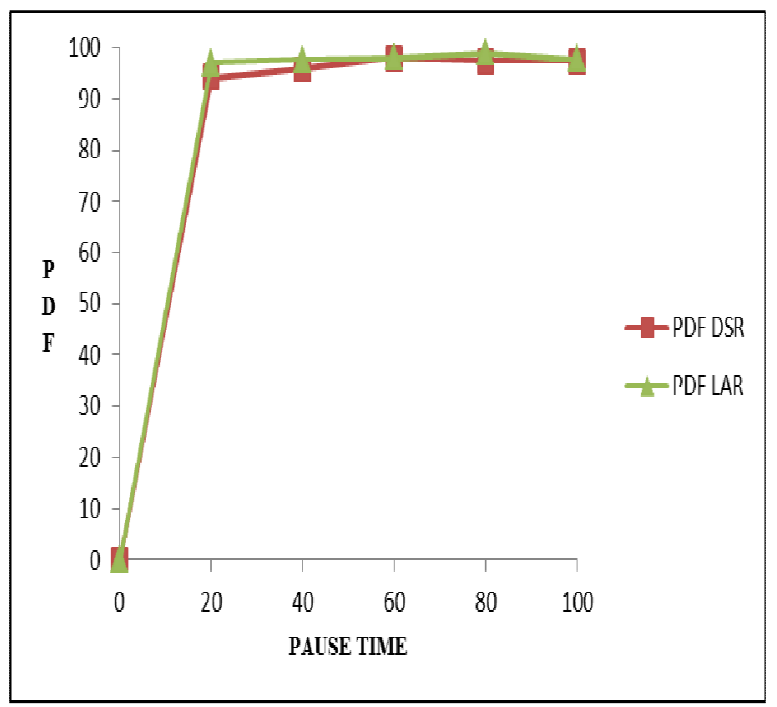

Figure.3 (a) PDF Vs Pause Time

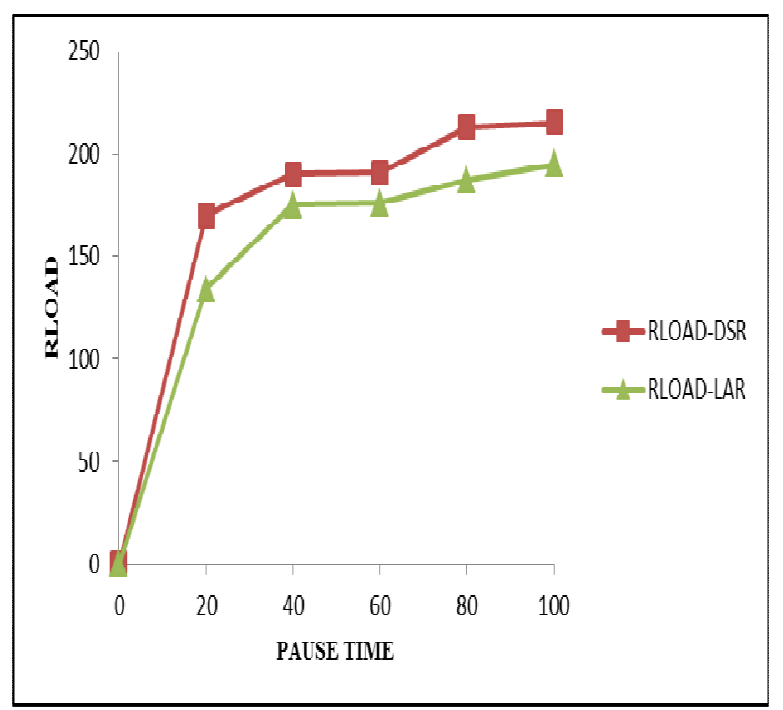

Fig.3 (b) Routing Overhead (RLOAD) Vs Pause Time

Fig.3 PDF and RLOAD variations $(n=12)$

\subsubsection{Results-}

After simulating in NS-2 value of PDF increases using LAR by $0.86 \%$, reduces routing overhead, NRL, E2E Delay and Dropped packets by 38\%, 40\%, 17.5\%, 30.2\% respectively compared to DSR protocol. 
International Journal of Wireless \& Mobile Networks (IJWMN) Vol. 4, No. 3, June 2012

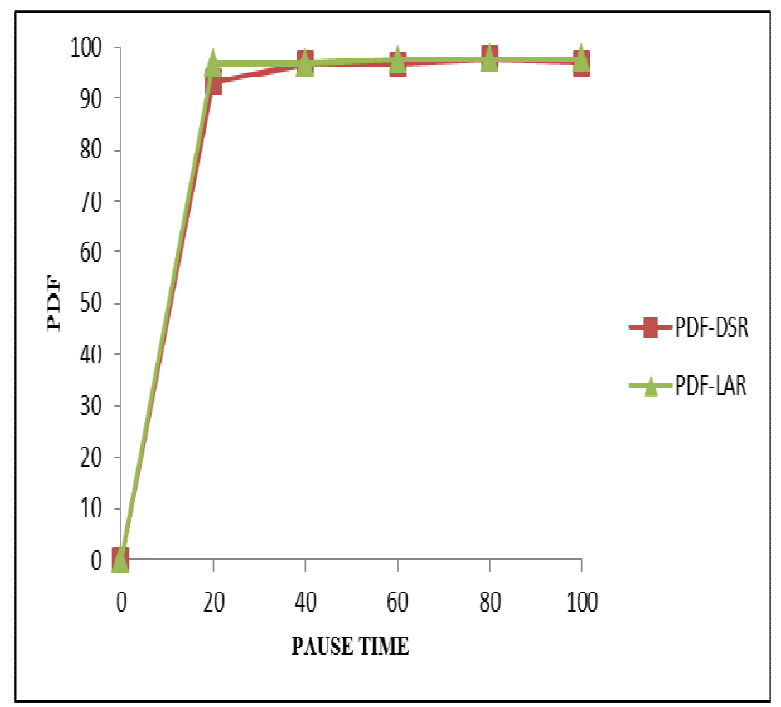

Fig.4 (a) PDF Vs Pause Time

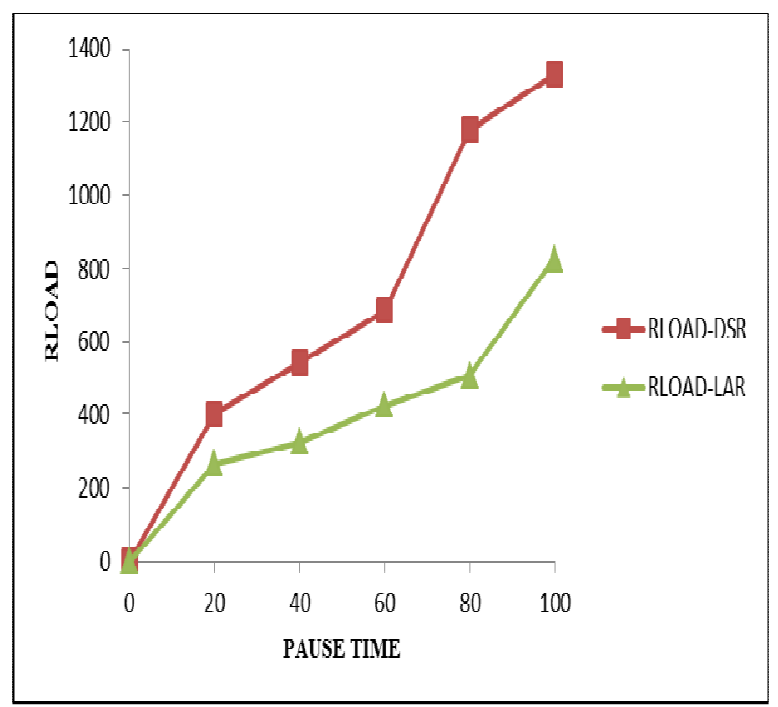

Fig.4 (b) RLOAD Vs Pause Time

Fig.4 PDF and RLOAD variations $(n=24)$

\subsubsection{Results-}

After simulating in NS-2 simulator value of PDF increases using LAR by $2.93 \%$, routing overhead, NRL, E2E Delay and Dropped packets by 22.5\%, 32.5\%, 47.75\%, $53.28 \%$ respectively compared to DSR protocol. 
International Journal of Wireless \& Mobile Networks (IJWMN) Vol. 4, No. 3, June 2012

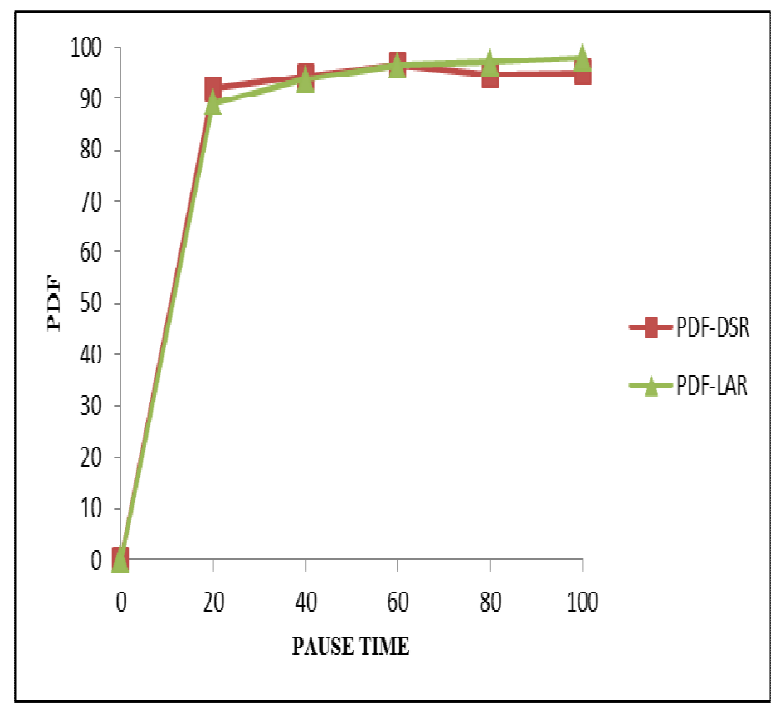

Fig.5 (a) PDF Vs Pause Time

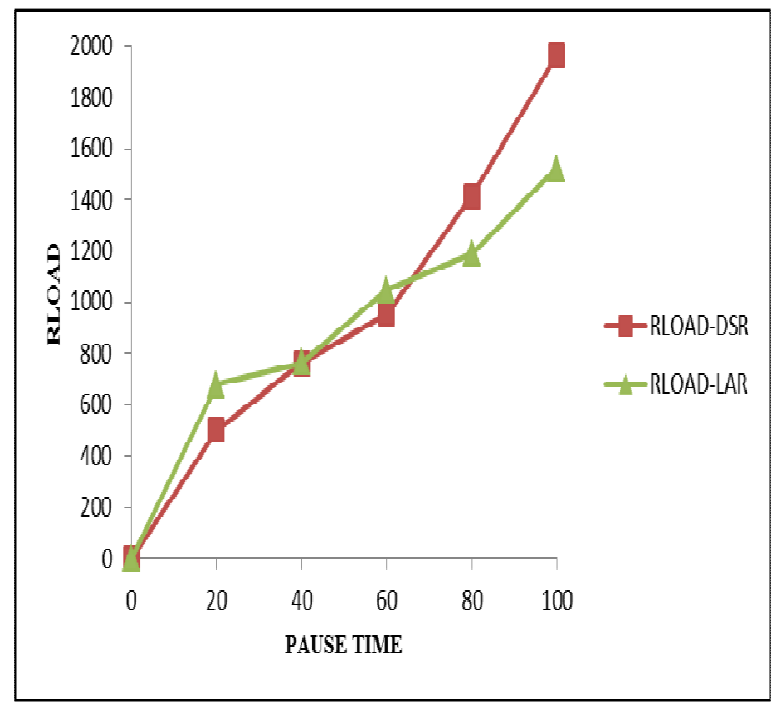

Fig.5 (b) RLOAD Vs Pause Time

Fig.5 PDF and RLOAD variations $(n=36)$

\subsubsection{Results-}

After simulating in NS-2 simulator value of PDF increases using LAR by $3.33 \%$ and reduces routing overhead, NRL, E2E Delay and Dropped packets by 3.25\%, 10.9\%, 26.68\%, 9.1\% respectively compared to DSR protocol. 


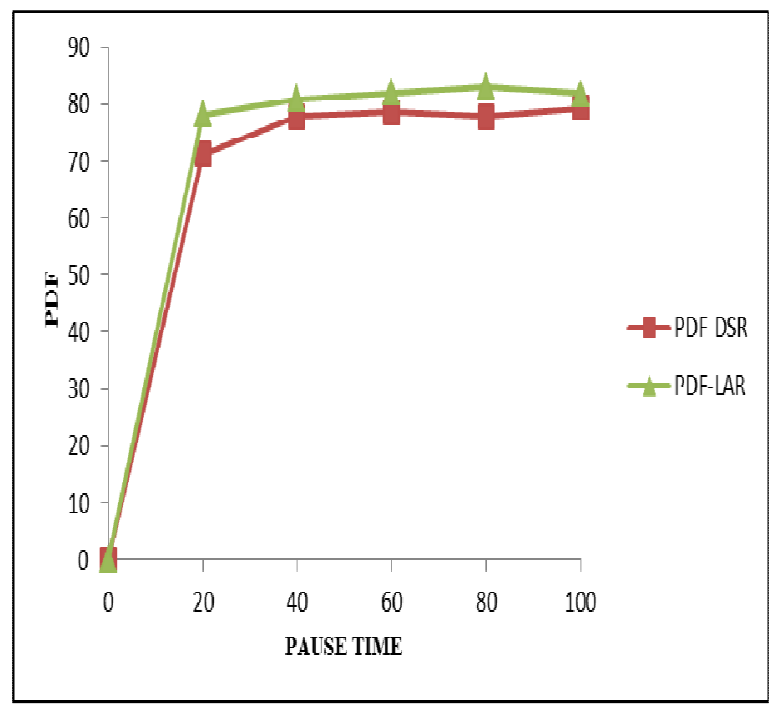

Figure.6 (a) PDF Vs Pause Time

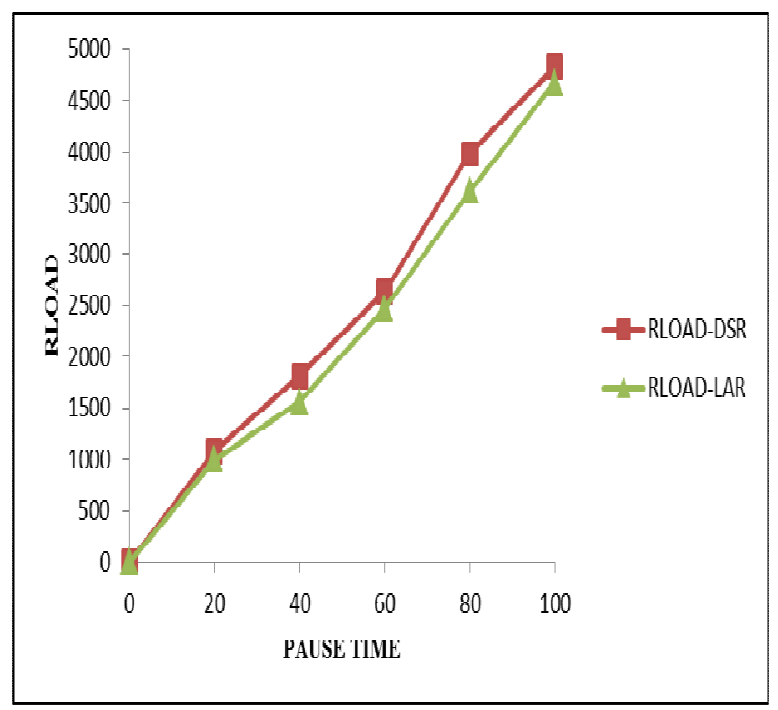

Figure.6 (b) RLOAD Vs Pause Time

Fig.6 PDF and RLOAD variations $(n=48)$

\subsubsection{Results-}

Next graph results are plotted between PDF versus Number of nodes and Routing Overhead versus Number of nodes. As the number of nodes increase into the network, PDF ratio using LAR increases in a better ratio compared to DSR protocol. Similarly routing overhead value using LAR reduces more compared to DSR protocol as the number of nodes increases into the network. Because of reduced routing overhead NRL and E2E is to be reduced. Along with optimization of these performance metrics number of lost packet is also to be reduced. 


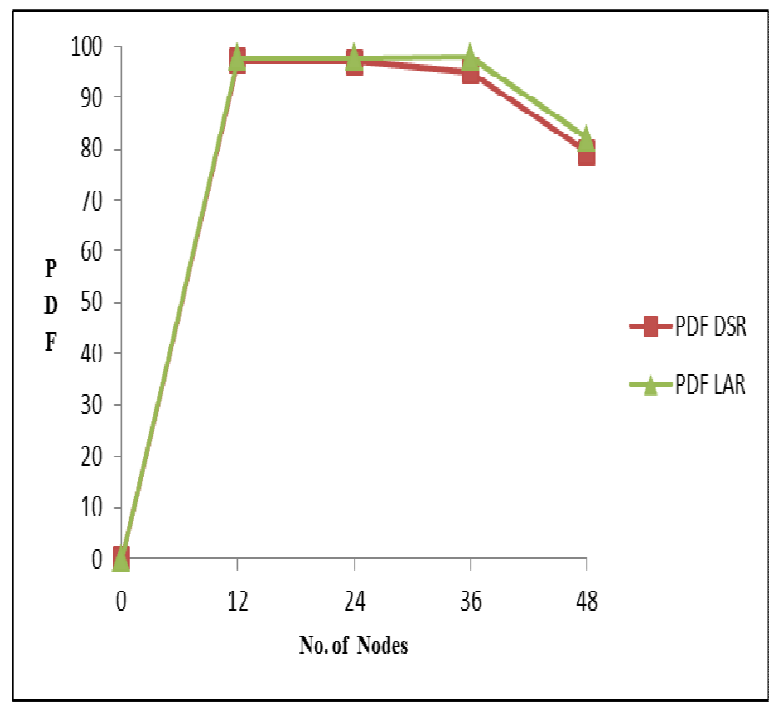

Figure.7 (a) PDF Vs Number of nodes

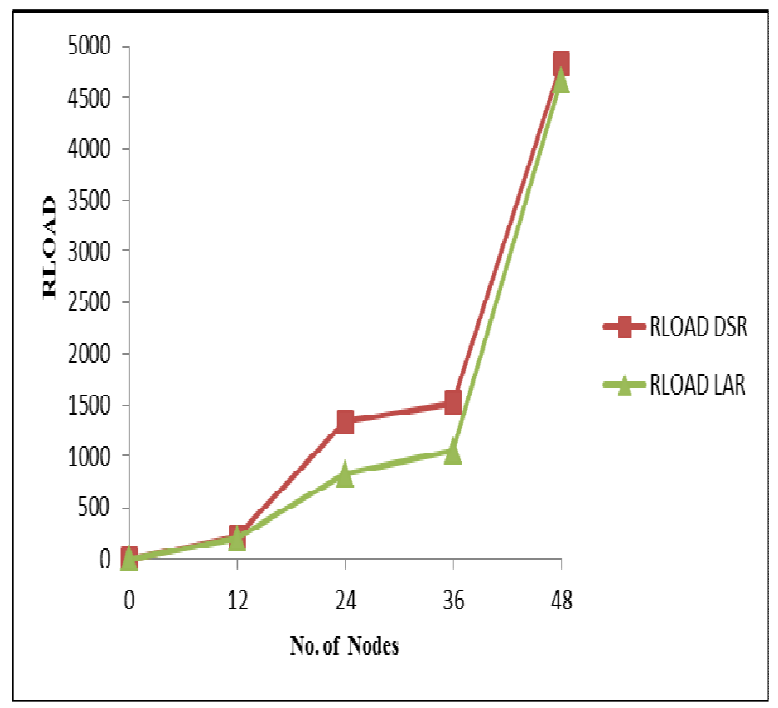

Fig.7 (b) RLOAD Vs Number of nodes

Figure.7 PDF and Routing Overhead (RLOAD) Vs Number of Nodes

\subsection{Under different fixed node mobility in MANET:}

In a dynamic Ad-Hoc network the mobility of nodes can not be ignored. It has a vital role in MANETs. Mobile nodes in the simulation travel according to the random-way point mobility model. Each simulation runs for 100 seconds. Simulation is run with movement patterns generated for $0,20,40,60,80$ and 100 pause times. NS-2 simulation is done at $5-20 \mathrm{~m} / \mathrm{s} \mathrm{mobility}$ 
International Journal of Wireless \& Mobile Networks (IJWMN) Vol. 4, No. 3, June 2012

speeds with same 12 numbers of sources in the networks. A result for the same is given in following figures of this section.

\subsubsection{Results:}

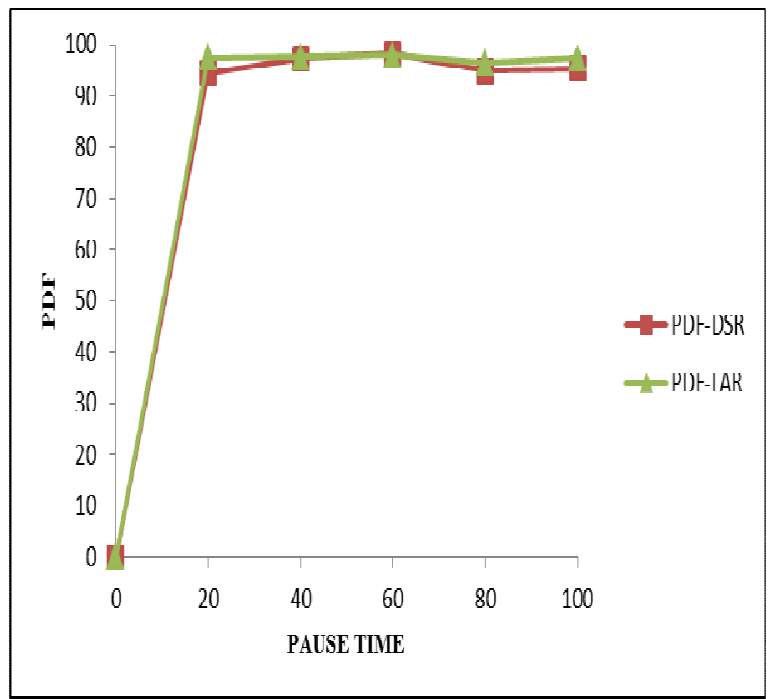

Figure.8 (a) PDF Vs Pause Time

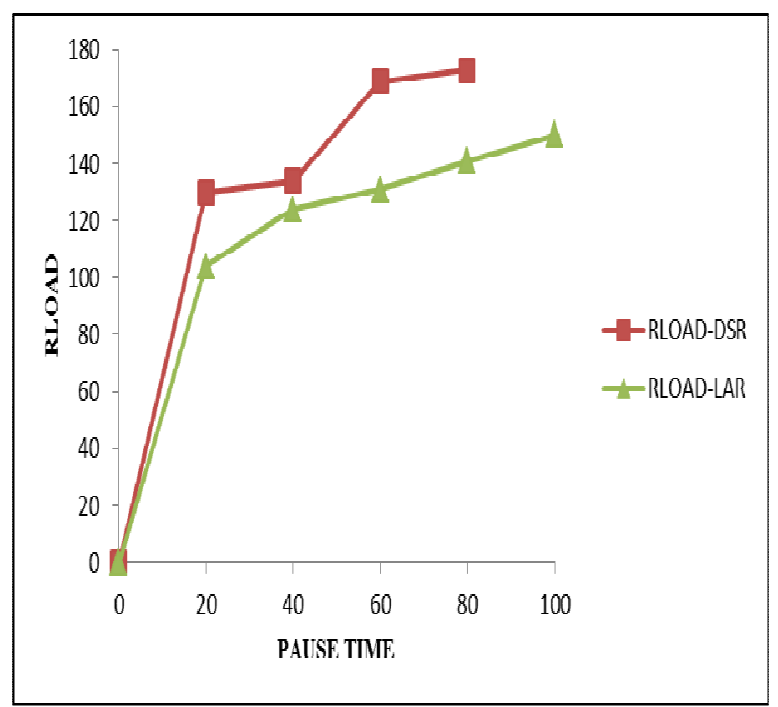

Fig.8 (b) RLOAD Vs Pause Time

Fig.8 PDF and RLOAD variations $(5 \mathrm{~m} / \mathrm{s})$ 


\subsubsection{Results:}

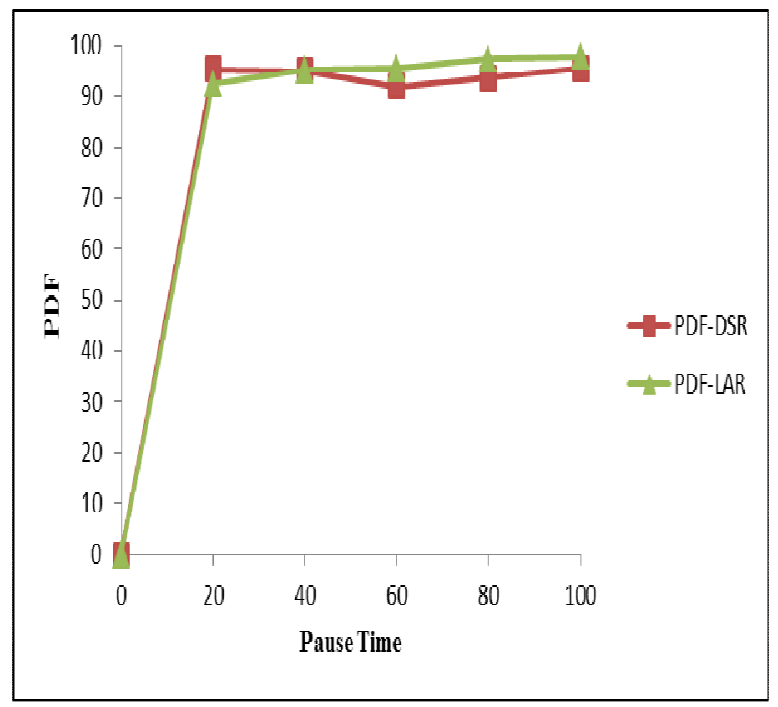

Fig.9 (a) PDF Vs Pause Time

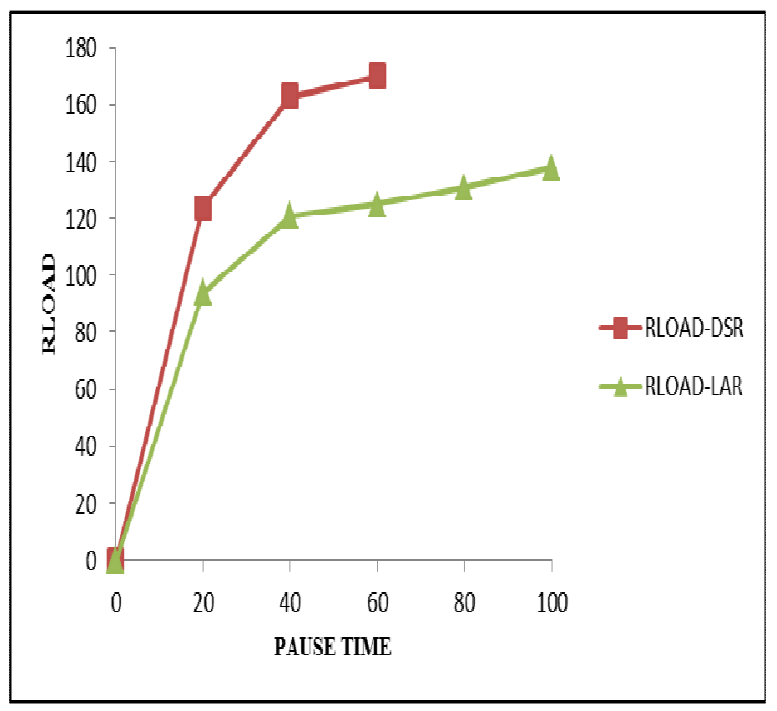

Fig.9 (b) RLOAD Vs Pause Time

Fig.9 PDF and RLOAD variations $(10 \mathrm{~m} / \mathrm{s})$ 


\subsubsection{Results:}

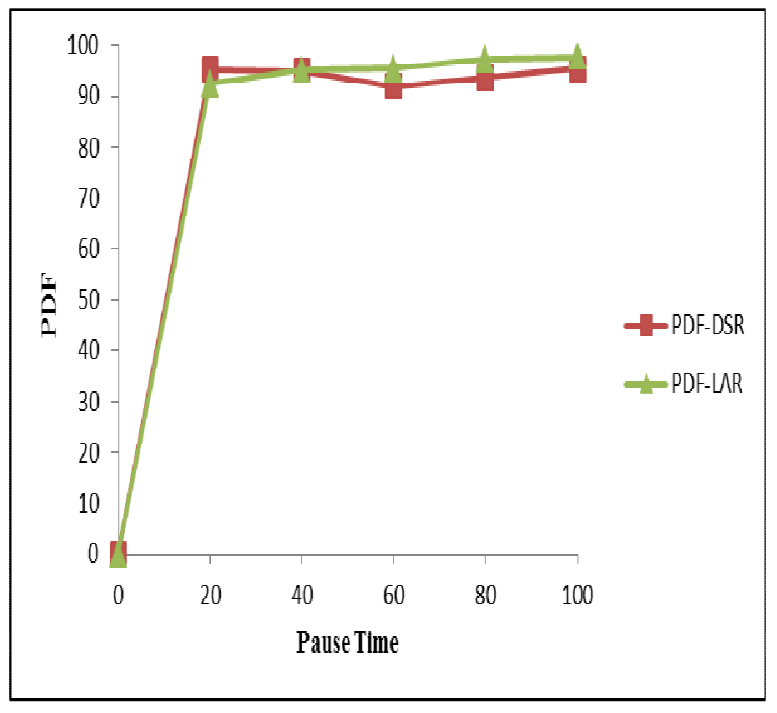

Fig. 10 (a) PDF Vs Pause Time

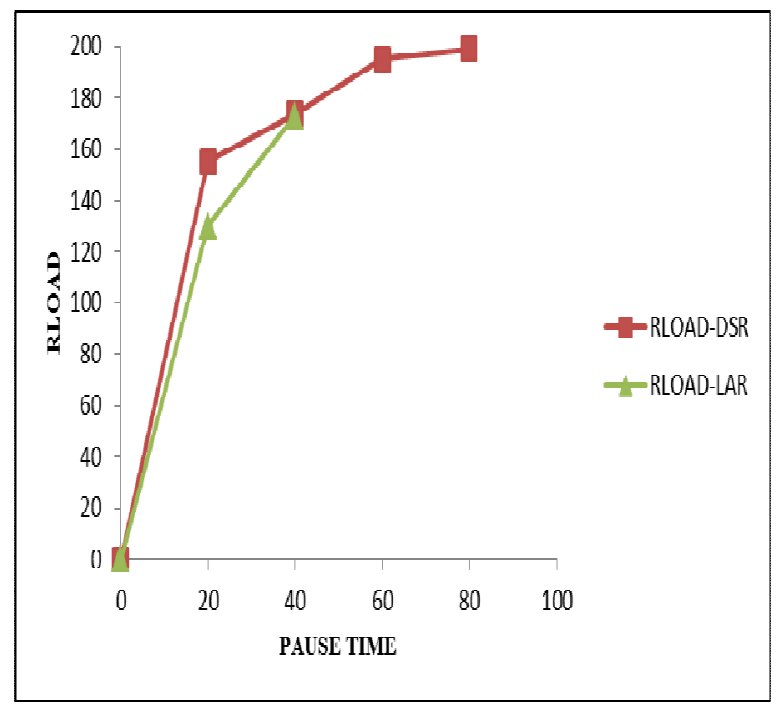

Fig.10 (b) RLOAD Vs Pause Time

Fig.10 PDF and RLOAD variations $(15 \mathrm{~m} / \mathrm{s})$ 


\subsubsection{Results:}

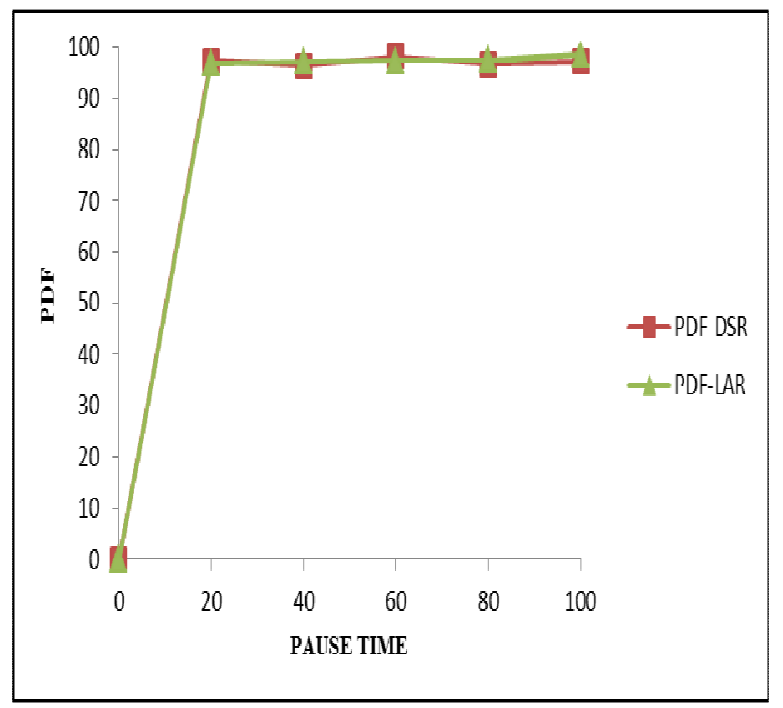

Fig. 11 (a) PDF Vs Pause Time

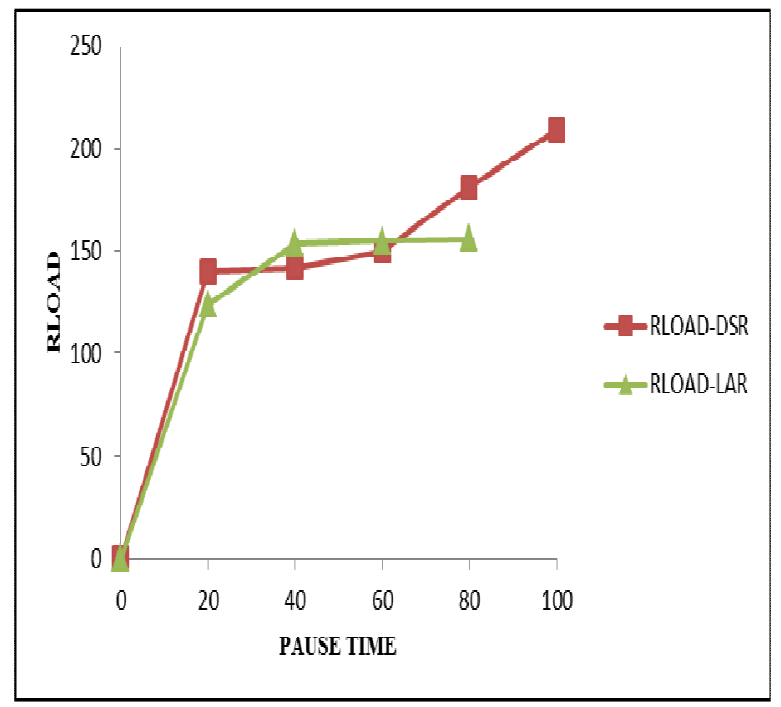

Fig.11 (b) RLOAD Vs Pause Time

Fig.11 PDF and RLOAD variations $(20 \mathrm{~m} / \mathrm{s})$

From the above results, it is found that performance metrics are improved in a better ratio in high density networks at varrying mobility, that is the main advantage of position-based LAR protocol. It has been concluded from the evaluted results that performace metrics of MANET are better in case of LAR compared to DSR whenever number of nodes increases in the networks that is basic aim of this paper. 
International Journal of Wireless \& Mobile Networks (IJWMN) Vol. 4, No. 3, June 2012

\section{CONCLUSION}

Ad-Hoc networks are characterized by multi-hop wireless connectivity and frequently changing network topology. There is a need for efficient dynamic routing protocols for MANETs; due to the scalability of the routing approach is an extremely essential. One of the approaches to scale up Ad-Hoc routing is geographical location-based routing (like a LAR). Because LAR floods only in the direction of expected destination istead of entire Ad-Hoc network. Hence routing overhead during LAR route discovery is to be minimized in other directions (except the direction of destination) and performance will be better in network. It is concluded that evalution of LAR protocol produces better results compared to DSR protocol in high density Ad-Hoc networks. Furthermore evalution of both was accomplished at varrying node mobility also in the same simultion scenerio. Finally it is concluded that LAR creats optimization of performance metrics such as, PDF, E2E Delay,routing overhead, NRL and lost data packets in MANETs. These optimization of parameters are important in case of Ad-Hoc network applications.

\section{REFERENCES}

[1] Broustis, I., Jakllari, G. Repantis, T.; Molle, M. "A Comprehensive Comparison of Routing Protocols for Large-Scale Wireless MANETs", 3rd Annual IEEE Communications Society on Sensor and Ad Hoc Communications and Networks (SECON '06), Vol.3, pp 951-956, Sept. 2006.

[2] Camp T., Boleng J., Williams B., Wilcox L., Navidi W., "Performance Comparison of Two Location Based Routing Protocols for Ad Hoc Networks", vol.3, pp 1678-1687, INFOCOM 2002.

[3] Das S.,Perkins C. and Royer E. "Performance Comparison of Two On-demand routing Protocols for Ad-Hoc network" Nineteenth Annual Joint Conference of the IEEE Computer and Communications Societies, IEEE INFOCOM, vol. 1,pp. 3-12, Tel Aviv, 2000.

[4] David Oliver Jörg "Performance Comparison of MANET Routing Protocols in Different Network Sizes" Computer Science Project, Institute of Computer Science and Applied Mathematics Computer Networks and Distributed Systems (RVS) University of Berne, Switzerland 2003.

[5] Dearham N., Quazi T. and McDonald S. “A Comparative Assessment of Ad-Hoc Routing Protocols" Proceedings of the South African Telecommunications Networks and Applications Conference (SATNAC), 2003.

[6] E. Ahvar, and M. Fathy, "Performance Evaluation of Routing Protocols for High Density AdHoc Networks based on Energy Consumption by GlomoSim Simulator" World Academy of Science, Engineering and Technology 292007.

[7] Gupta N. and Gupta R., "Routing Protocols in Mobile Ad-Hoc Network: An Overview” Proc. International Conference on Emerging Trends in Robotics and Communication Technologies (INTERACT), IEEE Explore pp. 173-177, 3-5 Dec., Chennai, 2010.

[8] Ko Y.B and Vaidya, N.H. "Location-Aided Routing (LAR) in Mobile Ad-Hoc Networks," Proc. IEEE MobiCom, Oct. 1998.

[9] Mauve M. Widmer A. and Hartenstein H. "A Survey on Position-Based Routing in Mobile Ad-Hoc Networks” IEEE Network, vol. 15, Issue 6, pp.30-39, Nov.2001.

[10] NS-2 Network simulator http://www.isi.edu/nsnam/ns.

[11] Qabajeh L. Mat Kiah L. Qabajeh M., “A Qualitative Comparison of Position-Based Routing Protocols for Ad-Hoc Networks" IJCSNS International Journal of Computer Science and Network Security, VOL.9 No.2, pp. 131-140, February 2009. 


\section{Authors:}

Neelesh Gupta is Pursuing Ph.D in Electronics and Communication from Rajiv Gandhi Technical University (RGTU), Bhopal (M.P.) India. He has a rich experience of teaching in various Technical institutions of reputed in MP, India. He is having more than 09 years of teaching Experience. Presently he is an Assistant Professor in Truba Institute of Engineering and Information Technology (T.I.E.I.T.), Bhopal (M.P.)-India. He has earned his MTech degree in Microwave and Millimeter Wave in 2007 from MANIT, Bhopal. His area of research Interests are Wireless Communication, Microwaves and Digital Signal Processing. He has presented a number of research papers in various National, International conferences and reputed International Journals. He is Life time member of IETE, New Delhi.

Dr. Roopam Gupta is currently working as an Associate Professor \& HOD in IT department, University Institute of Technology (UIT), RGPV, Bhopal (M.P.)-India. She received her Ph.D from Barkatullah University, Bhopal and M.Tech from MANIT, Bhopal (M.P.)-India. She has nineteen years of teaching experience. Her research interests are in the areas of Communication and Computer Networking. She is the recipient of MPCST, Young Scientist Award; 1999.She is Life Member of IETE and ISTE.

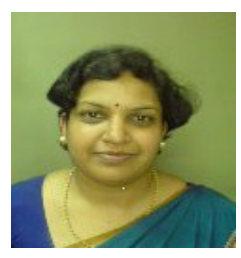

